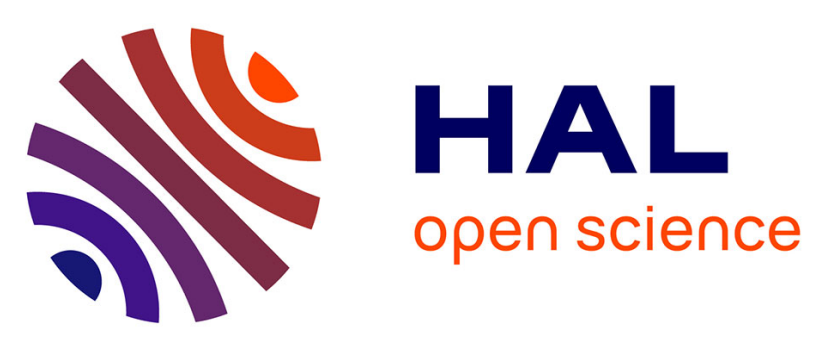

\title{
Optimal Consumption and Portfolio in a Jump Diffusion Market with Proportional Transaction Costs
}

Nils Chr. Framstad, Bernt Oksendal, Agnès Sulem

\section{To cite this version:}

Nils Chr. Framstad, Bernt Oksendal, Agnès Sulem. Optimal Consumption and Portfolio in a Jump Diffusion Market with Proportional Transaction Costs. [Research Report] RR-3749, INRIA. 1999. inria-00072913

\section{HAL Id: inria-00072913 \\ https://hal.inria.fr/inria-00072913}

Submitted on 24 May 2006

HAL is a multi-disciplinary open access archive for the deposit and dissemination of scientific research documents, whether they are published or not. The documents may come from teaching and research institutions in France or abroad, or from public or private research centers.
L'archive ouverte pluridisciplinaire HAL, est destinée au dépôt et à la diffusion de documents scientifiques de niveau recherche, publiés ou non, émanant des établissements d'enseignement et de recherche français ou étrangers, des laboratoires publics ou privés. 


\title{
Optimal consumption and portfolio in a jump diffusion market with proportional transaction costs
}

\author{
Nils Chr. Framstad, Bernt $\varnothing$ ksendal and Agnès Sulem
}

No 3749

Août 1999

THÈME 4

apport

de recherche 



\title{
Optimal consumption and portfolio in a jump diffusion market with proportional transaction costs
}

\author{
Nils Chr. Framstad*, Bernt Øksendal ${ }^{\dagger}$ and Agnès Sulem ${ }^{\ddagger}$ \\ Thème 4 - Simulation et optimisation \\ de systèmes complexes \\ Projet Mathfi
}

Rapport de recherche $\mathrm{n}^{\circ} 3749$ - Août 1999 - 24 pages

\begin{abstract}
We study the optimal consumption and portfolio in a jump diffusion market with proportional transaction costs. We show that the solution in the jump diffusion case has the same form as in the pure diffusion case; in particular, (under some assumptions) there is a no transaction cone $D$ such that it is optimal to make no transactions as long as the wealth position remains in $D$ and to sell/buy stocks according to local time on the boundary of $D$. The associated integrodifferential variational inequality is studied by using the theory of viscosity solutions.
\end{abstract}

Key-words: Singular stochastic control, Lévy processes, Reflected diffusion processes, Viscosity solution, Integro-differential Variational Inequalities, Portfolio selection, Transaction costs.

(Résumé : tsvp)

* Dep. of Mathematics, University of Oslo, Box 1053 Blindern, N-0316 Oslo, Norway, Email: ncf@math.uio.no

$\dagger$ Dep. of Mathematics, University of Oslo, and Norwegian School of Economics and Business Administration, Helleveien 30,N-5035 Bergen-Sandviken, Norway, Email: oksendal@math.uio.no

$\ddagger$ Email: Agnes.Sulem@inria.fr 


\section{Optimisation de portefeuilles et consommation optimale dans le cas de diffusions avec sauts en présence de coûts de transaction}

Résumé : On étudie la politique optimale d'investissement et de consommation d'un agent possédant un actif non risqué et un actif risqué modélisé par un processus de diffusion avec saut, dans le cas de coûts de transaction proportionnels. On montre que la solution a la même forme que dans le cas d'une diffusion pure traité par Davis and Norman [DN]. On montre en particulier qu'il existe sous certaines hypothèses un cône de non transaction $D$ où il est optimal de ne faire aucume transaction tant que la position de l'investisseur s'y trouve et d'acheter et de vendre selon des temps locaux sur la frontière de $D$. On établit l'inéquation variationnelle intégro-différentielle associée à ce problème que l'on étudie par la théorie des solutions de viscosité.

Mots-clé : Contrôle stochastique singulier, Processus de diffusion réfléchis, Solution de viscosité, Inéquation variationelle, Equations intégro-différentielles, Gestion de portefeuille, Coûts de transaction. 


\section{Introduction}

In this paper we study the problem of optimal consumption and investment policy in a jump diffusion market consisting of a bank account and a stock, whose price is modeled by a geometric Lévy process. Suppose the bank account gives a fixed interest rate $r$. Then the price $P_{1}(t)$ of this asset is given by

$$
d P_{1}(t)=r P_{1}(t) d t, \quad P_{1}(0)=p_{1}>0
$$

Let $P_{2}(t)$ denote the price of the stock at time $t$. Assume that $P_{2}(t)$ is a càdlàg process satisfying the following stochastic differential equation

$$
d P_{2}(t)=\alpha P_{2}(t) d t+\sigma P_{2}(t) d W(t)+P_{2}\left(t^{-}\right) \int_{-1}^{\infty} \eta \tilde{N}(d t, d \eta) ; \quad P_{2}(0)=p_{2}>0 .
$$

Here $\alpha \geq r$ and $\sigma>0$ are constant, $W(t)$ is a Wiener process on a filtered probability space $\left(\Omega, \mathcal{F}, \mathcal{F}_{t}, P\right)$ and

$$
\tilde{N}(t, A)=N(t, A)-t q(A) ; \quad t \geq 0, A \in \mathcal{B}(-1, \infty)
$$

is the compensator of a homogeneous Poisson random measure $N(t, A)$ on $\mathbb{R}^{+} \times \mathcal{B}(-1, \infty)$ with intensity measure $\mathbb{E}[N(t, A)]=t q(A)$, where $q$ is the Lévy measure associated to $N$ and $\mathcal{B}(-1, \infty)$ denotes the Borel $\sigma$-algebra on $(-1, \infty)$. See e.g. Bensoussan and Lions [BL], Jacod and Shiryaev [JS] and Protter [P] for more information about such stochastic differential equations. We assume that

$$
\|q\|:=q((-1, \infty))<\infty .
$$

Note that since we only allow jump sizes $\eta$ which are bigger than -1 , the process $P_{1}(t)$ will remain positive for all $t \geq 0$, a.s.

We assume that at any time $t$ the investor can choose a rate $c(t)$ that we can transfer money at any time from one asset to the other with a transaction cost which is proportional to the size of the transaction. Let $X(t), Y(t)$ denote the amount of money invested in asset number 1,2 , respectively. Then the evolution equations for $X(t), Y(t)$ are

$$
\begin{aligned}
d X(t) & =d X^{c, \mathcal{L}, \mathcal{M}}(t) \\
& =(r X(t)-c(t)) d t-(1+\lambda) d \mathcal{L}(t)+(1-\mu) d \mathcal{M}(t) ; \quad X\left(0^{-}\right)=x \in \mathbb{R} \\
d Y(t) & =d Y^{\mathcal{L}, \mathcal{M}}(t) \\
& =Y\left(t^{-}\right)\left(\alpha d t+\sigma d W(t)+\int_{-1}^{\infty} \eta \tilde{N}(d t, d \eta)\right)+d \mathcal{L}(t)-d \mathcal{M}(t) ; \quad Y\left(0^{-}\right)=y \in \mathbb{R} .
\end{aligned}
$$

Here $\mathcal{L}(t), \mathcal{M}(t)$ represent cumulative purchase and sale, respectively, of stocks up to time $t$. The coefficients $\lambda \geq 0, \mu \in[0,1]$ represent the constants of proportionality of the transaction costs. We have to make a choice of version of the processes. We can either let $\mathcal{L}$ and $\mathcal{M}$ be left-continuous, which is maybe the natural choice from an impulse control point of view; or we can stick to the usual set-up of stochastic integration and let all processes be right-continuous. We will choose the latter approach. 
Remark 1.1. By multiplying all processes by $e^{-r t}$ and differentiating using the Itô formula, one will see that the problem only depends on $\alpha$ and $r$ through their difference, just like the Merton problem. It would in fact suffice to consider the case $r=0$; In this case, $X(t)$ would be nonincreasing except at the times we sell stocks.

Our controls will have to meet certain conditions. The solvency region $\mathcal{S}$ is defined to be the set of states where the net wealth is nonnegative:

$$
\mathcal{S}=\left\{(x, y) \in \mathbb{R}^{2} ; x+(1+\lambda) y \geq 0 \text { and } x+(1-\mu) y \geq 0\right\}
$$

with boundaries $\partial_{1} \mathcal{S}, \partial_{2} \mathcal{S}$ as in Figure 1.

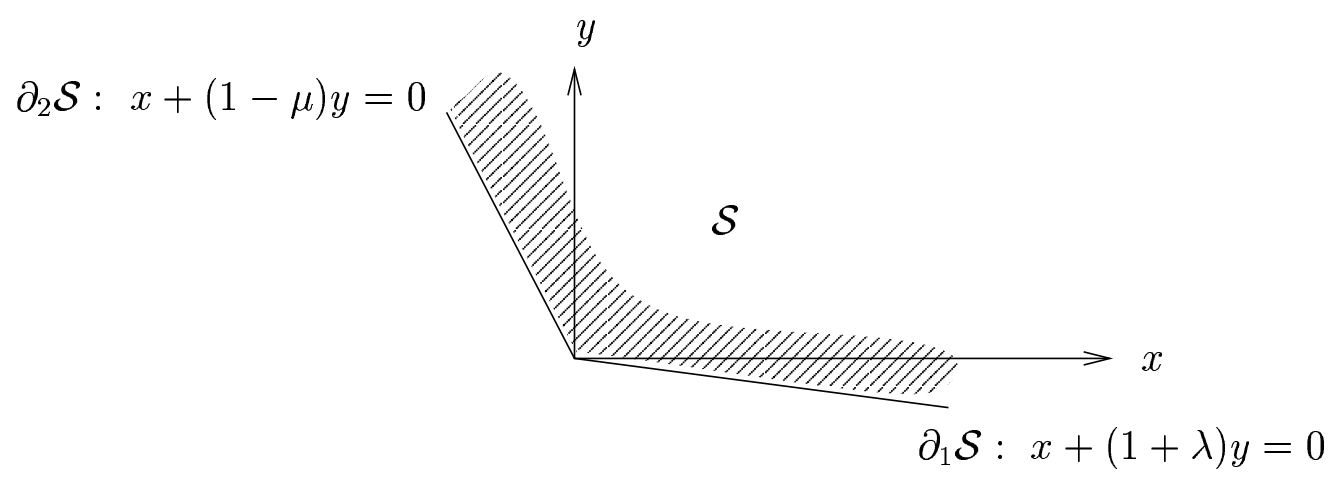

Figure 1: The solvency region

It is natural to require that

$$
\text { If }(x, y) \in \mathcal{S} \text {, then }(X(t), Y(t)) \in \mathcal{S} \text { for all } t \text { (a.s.). }
$$

Note that in the presence of a jump term, we need to make sure that we can cover any position we (with nonzero probability) could happen to jump to. Hence, if we define $U^{\prime} \subset \mathcal{S}$ as

$$
U^{\prime}=\{(x, y) ;(x, y(1+\eta)) \in \mathcal{S} \text { for all } \eta \in \operatorname{supp} q\}
$$

then it is necessary and sufficient for (1.6) to hold that

$$
\text { If }(x, y) \in \mathcal{S} \text {, then }(X(t), Y(t)) \in \overline{U^{\prime}} \text { for all } t \text { (a.s.). }
$$

(Note that had we not chosen the right continuous version, we should have weakened the condition to a.a. $t$.) Since we already have to deal with a cone contained in $\mathcal{S}$ (with equality iff $q=0$ ), we get the following generalization more or less for free: Let $U \subseteq U^{\prime}$ be a given open convex cone with vertex at the origin. It will later be convenient to characterize $U$ in terms of polar coordinates; let $\partial U$ be given by angles $\theta_{1} \in\left[-\frac{\pi}{2}, \frac{3 \pi}{2}\right)$ and $\theta_{2} \in\left(\theta_{1}, \frac{3 \pi}{2}\right]$ (and such that $U \subseteq U^{\prime}$ ). Thus,

$$
U=\left\{(x, y)=R e^{i \theta} ; \quad R \geq 0 ; \quad \theta_{1}<\theta<\theta_{2}\right\} .
$$

Then we will require the following:

$$
\text { If }(x, y) \in \mathcal{S} \text {, then }(X(t), Y(t)) \in \bar{U} \text { for all } t \text { (a.s.) }
$$


The restriction to a (possibly) smaller cone $U$ may be given an economic interpretation as (say, law enforced) restrictions on short sale or leverage. Of particular interest is the case where $U$ is the first quadrant. This serves as the authors' "moral justification" for the restrictive assumption of Theorem 4.3, that the no transaction region is contained in the first quadrant (eq.(4.9)) - an assumption we conjecture not to hold if the Merton line lies outside the first quadrant.

Definition 1.2. The set $\mathcal{A}$ of admissible controls is the set of consumption-investment policies $(c(t), \mathcal{L}(t), \mathcal{M}(t))$ satisfying the following:

(i) The processes $c(t), \mathcal{L}(t), \mathcal{M}(t)$ are predictable.

(ii) $c(t, \omega) \geq 0$ for a.e. $(t, \omega)$.

(iii) $\mathcal{L}(t), \mathcal{M}(t)$ are right-continuous, non-decreasing and $\mathcal{L}\left(0^{-}\right)=\mathcal{M}\left(0^{-}\right)=0$.

(iv) Equation (1.9) holds.

The intuitive interpretation of (iv) above, is that if a jump should bring us out of $\bar{U}$, then an admissible control will bring us back into $\bar{U}$ immediately. Now since we have chosen to work with the right-continuous version, then "out of $\bar{U}$ " should be interpreted as

$$
\left(X\left(t^{-}\right), Y\left(t^{-}\right)+\Delta_{N} Y(t)\right) \notin \bar{U}
$$

where

$$
\Delta_{N} Y(t):=Y\left(t^{-}\right) \int_{-1}^{\infty} \eta N(\{t\}, d \eta)
$$

and $N(\{t\}, \cdot)$ denotes the jump in the Poisson random measure occuring at time $t$. Define the performance criterion by

$$
J^{c, \mathcal{L}, \mathcal{M}}(x, y)=\mathbb{E}^{x, y}\left[\int_{0}^{\infty} e^{-\delta t} \frac{c^{\gamma}(t)}{\gamma} d t\right]
$$

where $\delta>0, \gamma \in(0,1)$ are constants and $\mathbb{E}^{x, y}$ is the expectation with respect to the probability law $P^{x, y}$ of $(X(t), Y(t))$ when $\left(X\left(0^{-}\right), Y\left(0^{-}\right)\right)=(x, y) \in \mathbb{R}^{2}$. The problem is to find $V$ and (if exists) $\left(c^{*}, \mathcal{L}^{*}, \mathcal{M}^{*}\right) \in \mathcal{A}$ such that

$$
V(x, y)=\sup _{(c, \mathcal{L}, \mathcal{M}) \in \mathcal{A}} J^{c, \mathcal{L}, \mathcal{M}}(x, y)=J^{c^{*}, \mathcal{L}^{*}, \mathcal{M}^{*}}(x, y) .
$$

In the special case when the stock price is a geometric Brownian motion (i.e., $q=0$ ) and there are no transaction costs (i.e. $\lambda=\mu=0$ ) this problem was first studied by Merton [M]. He proved that if

$$
\delta>\gamma\left[r+\frac{(\alpha-r)^{2}}{2 \sigma^{2}(1-\gamma)}\right]
$$

then the value function $V_{0}(x, y)$ is given by

$$
V_{0}(x, y)=K_{0}(x+y)^{\gamma}
$$


where

$$
K_{0}=\frac{1}{\gamma}\left[\frac{1}{1-\gamma}\left(\delta-\gamma r-\frac{\gamma(\alpha-r)^{2}}{2 \sigma^{2}(1-\gamma)}\right)\right]^{\gamma-1} .
$$

Moreover, the corresponding optimal consumption $c_{0}^{*}$ is given (in feedback form) by

$$
c_{0}^{*}(x, y)=\left(K_{0} \gamma\right)^{\frac{1}{\gamma-1}}(x+y)
$$

and the corresponding optimal portfolio is to keep the fraction $Y(t) /(X(t)+Y(t))$ of wealth invested in the stocks constantly equal to the value

$$
u_{0}^{*}=\frac{\alpha-r}{(1-\gamma) \sigma^{2}}
$$

at all times. In other words, it is optimal to perform transactions in such a way that the state $(X(t), Y(t))$ is always situated on the line $y=\frac{u_{0}^{*}}{1-u_{0}^{*}} x$ in the $(x, y)$-plane (the Merton line).

In $[\mathrm{K}]$ and later in $[\mathrm{F} \varnothing \mathrm{S}]$ the results of Merton are extended to the case when the stock price is a geometric Lévy process (i.e., given by (1.2)), still assuming that there are no transaction costs, i.e., $\lambda=\mu=0$. It is proved that the value function $V(x, y)$ still has the same form, namely

$$
V(x, y)=K(x+y)^{\gamma}
$$

but with a different constant $K$ (under an assumption similar to (1.13)). The corresponding optimal consumption $c^{*}$ is given by

$$
c^{*}(x, y)=(K \gamma)^{\frac{1}{\gamma-1}} \cdot(x+y)
$$

and the optimal portfolio is to keep the fraction $Y(t) /(X(t)+Y(t))$ constantly equal to a value $u^{*}$. See [FØS, Theorem 2.3]. In [FØS, Corollary 2.4] it is proved that if $q \neq 0$ then

$$
\begin{aligned}
& V(x, y)<V_{0}(x, y), \\
& c^{*}(x, y)>c_{0}^{*}(x, y)
\end{aligned}
$$

and

$$
u^{*}<u_{0}^{*}
$$

In fact, the introduction of the jump term involving the integral with respect to $\tilde{N}$ has the same effect on the solution as increasing the volatility $\sigma$.

The purpose of this paper is to study the general case with the stock price given by a geometric Lévy process (1.2) and with proportional transaction costs. We prove that in this case there exists a no transaction region $D$ in the $(x, y)$-plane with the shape of a cone with vertex at the origin, such that it is optimal to make no transactions as long as $(X(t), Y(t)) \in D$ and to sell stocks at the rate of local time (of the reflected process) at the upper/left boundary of $D$ and purchase stocks at the rate of local time at the lower/right boundary. These results generalize the results of Davis and Norman $[\mathrm{DN}]$ who obtained similar results in the no jump case $(q=0)$. Our paper is also inspired by the paper of Shreve and Soner [SS], who also considered the case $q=0$. They used, as we do, a viscosity solution approach and were able to remove some of the assumptions in [DN]. Viscosity solutions of combined stochastic control and optimal stopping problems for jump diffusion processes are studied by $\mathrm{Pham}[\mathrm{Ph}]$. However, his conditions are not satisfied in the case we consider because our utility rate $\frac{c^{\gamma}}{\gamma}$ is not bounded as a function of $c \geq 0$. 


\section{An integro-variational inequality verification theorem}

In this section we show how to associate an integro-variational inequality to the value function $V(x, y)$ defined by (1.12).

First note that is we apply a Markov control $c(t)=c(X(t), Y(t))$ and there are no transactions, then the generator $\tilde{A}$ of the time-space process

$$
d \tilde{Z}(t)=(d t, d X(t), d Y(t))
$$

is given by

$$
\begin{aligned}
\tilde{A} \varphi(s, x, y)= & \frac{\partial \varphi}{\partial s}+(r x-c) \frac{\partial \varphi}{\partial x}+\alpha y \frac{\partial \varphi}{\partial y}+\frac{1}{2} \sigma^{2} y^{2} \frac{\partial^{2} \varphi}{\partial y^{2}} \\
& +\int_{-1}^{\infty}\left[\varphi(s, x, y+y \eta)-\varphi(s, x, y)-\frac{\partial \varphi}{\partial y}(s, x, y) \cdot y \eta\right] d q(\eta) .
\end{aligned}
$$

If $\varphi$ has the form

$$
\varphi(s, x, y)=e^{-\delta s} \psi(x, y)
$$

then

$$
\tilde{A} \varphi(s, x, y)=e^{-\delta s} A^{c} \psi(x, y)
$$

where

$$
\begin{aligned}
A^{c} \psi(x, y)= & -\delta \psi(x, y)+(r x-c) \frac{\partial \varphi}{\partial x}+\alpha y \frac{\partial \psi}{\partial y}+\frac{1}{2} \sigma^{2} y^{2} \frac{\partial^{2} \psi}{\partial y^{2}} \\
& +\int_{-1}^{\infty}\left[\psi(x, y+y \eta)-\psi(x, y)-\frac{\partial \psi}{\partial y}(x, y) \cdot y \eta\right] d q(\eta)
\end{aligned}
$$

If $(c(t), \mathcal{L}(t), \mathcal{M}(t))$ is an admissible control, we will in the following let $t_{k}$ denote the jumping times of $(\mathcal{L}(t), \mathcal{M}(t))$. The jumps of $\mathcal{L}(t), \mathcal{M}(t)$ at $t=t_{k}$ are

$$
\Delta \mathcal{L}\left(t_{k}\right)=\mathcal{L}\left(t_{k}\right)-\mathcal{L}\left(t_{k}^{-}\right), \quad \Delta \mathcal{M}\left(t_{k}\right)=\mathcal{M}\left(t_{k}\right)-\mathcal{M}\left(t_{k}^{-}\right),
$$

respectively. And we let

$$
\mathcal{L}_{c}(t):=\mathcal{L}(t)-\sum_{0 \leq t_{k} \leq t} \Delta \mathcal{L}\left(t_{k}\right), \quad \mathcal{M}_{c}(t):=\mathcal{M}(t)-\sum_{0 \leq t_{k} \leq t} \Delta \mathcal{M}\left(t_{k}\right)
$$

be the continuous part of $\mathcal{L}(t), \mathcal{M}(t)$, respectively. If $v$ is a continuous real function on $\mathcal{S}$ we let

$$
\Delta_{\mathcal{L}, \mathcal{M}} v\left(Z\left(t_{k}\right)\right)=v\left(Z\left(t_{k}\right)\right)-v\left(Z\left(t_{k}^{-}\right)\right)
$$

denote the jump in the value of $v(Z(t))$ caused by the jump of $(\mathcal{L}(t), \mathcal{M}(t))$ at $t=t_{k}$. We emphasize that the possible jumps in $Z(t)$ coming from $\tilde{N}$ are not included in $\Delta_{\mathcal{L}, \mathcal{M}} v\left(Z\left(t_{k}\right)\right)$.

First, we give a few properties of the value function:

Lemma 2.1. a) $V(x, y)$ is a nondecreasing function with respect to both $x$ and $y$. 
b) $V(x, y)$ is a concave function of $(x, y)$.

c) $V(x, y)$ is homogenous of degree $\gamma$, i.e. $V(\rho x, \rho y)=\rho^{\gamma} V(x, y)$ for all $\rho>0$.

d) Outside $U$, the value function has the form

$$
\begin{array}{ll}
V(x, y)=K_{1} \cdot(x+(1+\lambda) y)^{\gamma} & \text { on } \theta<\theta_{1}, \text { and } \\
V(x, y)=K_{2} \cdot(x+(1-\mu) y)^{\gamma} & \text { on } \theta>\theta_{2} .
\end{array}
$$

Proof. a) If $\bar{x} \geq x$ and $\bar{y} \geq y$, then one can reach $(x, y)$ from $(\bar{x}, \bar{y})$ by an immediate transaction (possibly both buying and selling at the same time).

b) This follows from the concavity of the utility function $c \mapsto \frac{1}{\gamma} c^{\gamma}$ and the linearity of (1.4), as in [AST, Prop. 3.1].

c) This follows as in [DN, Th.3.1a)].

d) Outside $\bar{U}$, a transaction (at least) to $\partial U$ is compulsory, and therefore the value of a state outside $U$ is constant along the half-lines from $U$ and parallell to the boundary of the solvency region. Then the claim follows from homogeneity.

Part d) above now characterizes the value function outside $U$.

Theorem 2.2 (Integro-variational inequality verification theorem). a) Suppose there exists a nonnegative function $v(x, y) \in C^{2}\left(\mathcal{S}^{0}\right)$ such that

$$
\begin{gathered}
L v:=-(1+\lambda) \frac{\partial v}{\partial x}+\frac{\partial v}{\partial y} \leq 0 \\
M v:=(1-\mu) \frac{\partial v}{\partial x}-\frac{\partial v}{\partial y} \leq 0 \\
A^{c} v+\frac{1}{\gamma} c^{\gamma} \leq 0 \text { on } U \text { for all } c \geq 0
\end{gathered}
$$

Then

$$
v(x, y) \geq V(x, y)
$$

b) Suppose, in addition to (2.4) - (2.6) that there exists $\hat{c}(x, y) \geq 0$ such that

$$
\max \left\{L v(x, y), A^{\hat{c}} v(x, y)+\frac{1}{\gamma} \hat{c}^{\gamma}(x, y), M v(x, y)\right\}=0
$$

for all $(x, y) \in U$. Define the no transaction region $D$ by

$$
D=\{(x, y) ; L v(x, y)<0 \text { and } M v(x, y)<0\}
$$


Suppose there exist $\hat{\mathcal{L}}(t), \hat{\mathcal{M}}(t)$ such that $\hat{w}:=(\hat{c}, \hat{\mathcal{L}}, \hat{\mathcal{M}})$ is admissible and such that, if we put

$$
Z^{\hat{w}}(t):=\left(X^{\hat{c}, \hat{\mathcal{L}}, \hat{\mathcal{M}}}(t), Y^{\hat{\mathcal{L}, \mathcal{M}}}(t)\right)
$$

then we have

$$
\begin{aligned}
& \left(-(1+\lambda) \frac{\partial v}{\partial x}+\frac{\partial v}{\partial y}\right)\left(Z^{\hat{w}}(t)\right) d \hat{\mathcal{L}}_{c}(t)=0 \text { for all } t \\
& \left((1-\mu) \frac{\partial v}{\partial x}-\frac{\partial v}{\partial y}\right)\left(Z^{\hat{w}}(t)\right) d \hat{\mathcal{M}}_{c}(t)=0 \text { for all } t
\end{aligned}
$$

where $\hat{\mathcal{L}}_{c}(t)=\hat{\mathcal{L}}(t)-\sum_{0 \leq t_{k} \leq t} \Delta \hat{\mathcal{L}}\left(t_{k}\right)$ is the continuous part of $\hat{\mathcal{L}}(t) ; \Delta \hat{\mathcal{L}}\left(t_{k}\right)=\hat{\mathcal{L}}\left(t_{k}\right)-\hat{\mathcal{L}}\left(t_{k}^{-}\right)$being the jump of $\hat{\mathcal{L}}(t)$ at the jumping times $t_{k}$ of $(\hat{\mathcal{L}}(t), \hat{\mathcal{M}}(t))$, and similarly with $\hat{\mathcal{M}}_{c}(t)$.

Moreover, suppose that (see (2.4)) for all jumping times $t_{k}$ of $(\hat{\mathcal{L}}(t), \hat{\mathcal{M}}(t))$ we have

$$
\Delta_{\mathcal{L}, \mathcal{M}} v\left(Z\left(t_{k}\right)\right):=v\left(Z\left(t_{k}\right)\right)-v\left(Z\left(t_{k}^{-}\right)\right)=0
$$

for all jumping times $t_{k}$ of $(\hat{\mathcal{L}}(t), \hat{\mathcal{M}}(t))$;

$$
Z^{\hat{w}}(t) \in \bar{D} \text { for almost all } t
$$

and

$$
\lim _{R \rightarrow \infty} \mathbb{E}^{x, y}\left[e^{-\delta T_{R}} v\left(Z^{\hat{w}}\left(T_{R}\right)\right)\right]=0
$$

where

$$
T_{R}=\min \left(R, \inf \left\{t>0 ;\left|Z^{\hat{w}}(t)\right| \geq R\right\}\right)
$$

Then

$$
v(x, y)=V(x, y) \text { for all }(x, y) \in U
$$

and the control $\hat{w}=(\hat{c}, \hat{\mathcal{L}}, \hat{\mathcal{M}})$ is optimal.

Proof. Several versions of this result are known. See e.g. [BL]. For completeness we include a sketch of a proof. If $v$ satisfies the conditions of a) then by the Ito formula for semimartingales (see e.g. [P, Th.II.7.33]) we have, for any admissible $(c, \mathcal{L}, \mathcal{M})$ with corresponding state process $Z(t)=(X(t), Y(t))$

$$
\begin{aligned}
\mathbb{E}^{x, y}\left[e^{-\delta T_{R}} v\left(Z\left(T_{R}\right)\right)\right]= & v(Z(0))+\mathbb{E}^{x, y}\left[\int_{0}^{T_{R}} e^{-\delta t} A^{c} v(Z(t)) d t\right] \\
& +\mathbb{E}^{x, y}\left[\int _ { 0 ^ { + } } ^ { T _ { R } } e ^ { - \delta t } \left\{\frac{\partial v}{\partial x}\left(Z\left(t^{-}\right)\right) \cdot(-(1+\lambda) d \mathcal{L}(t)+(1-\mu) d \mathcal{M}(t))\right.\right. \\
& \left.\left.+\frac{\partial v}{\partial y}\left(Z\left(t^{-}\right)\right) \cdot(d \mathcal{L}(t)-d \mathcal{M}(t))\right\}\right] \\
+ & \mathbb{E}^{x, y}\left[\sum _ { 0 < t _ { k } \leq T _ { R } } e ^ { - \delta t _ { k } } \left\{\Delta_{\mathcal{L}, \mathcal{M}} v\left(Z\left(t_{k}\right)\right)\right.\right. \\
& \left.\left.-\frac{\partial v}{\partial x}\left(Z\left(t_{k}^{-}\right)\right) \cdot \Delta_{\mathcal{L}, \mathcal{M}} X\left(t_{k}\right)-\frac{\partial v}{\partial y}\left(Z\left(t_{k}^{-}\right)\right) \cdot \Delta_{\mathcal{L}, \mathcal{M}} Y\left(t_{k}\right)\right\}\right]
\end{aligned}
$$


where $t_{k}$ denotes the times of jumps for $(\mathcal{L}(t), \mathcal{M}(t)), \Delta_{\mathcal{L}, \mathcal{M}} X\left(t_{k}\right)=X\left(t_{k}\right)-X\left(t_{k}^{-}\right)$and similarly for $\Delta_{\mathcal{L}, \mathcal{M}} Y\left(t_{k}\right), \Delta \mathcal{L}\left(t_{k}\right), \Delta \mathcal{M}\left(t_{k}\right)$ and $\Delta_{\mathcal{L}, \mathcal{M}} v\left(Z\left(t_{k}\right)\right):=v\left(Z\left(t_{k}\right)\right)-v\left(Z\left(t_{k}^{-}\right)\right)$as in (2.4). This can be written

$$
\begin{aligned}
\mathbb{E}^{x, y}\left[e^{-\delta T_{R}} v\left(Z\left(t_{R}\right)\right)\right]= & v(x, y)+\mathbb{E}^{x, y}\left[\int_{0}^{T_{R}} e^{-\delta t} A^{c} v(Z(t)) d t\right] \\
& +\mathbb{E}^{x, y}\left[\int_{0}^{T} e^{-\delta t} \cdot\left(-(1+\lambda) \frac{\partial v}{\partial x}+\frac{\partial v}{\partial y}\right)(Z(t)) d \mathcal{L}_{c}(t)\right] \\
& +\mathbb{E}^{x, y}\left[\int_{0}^{T} e^{-\delta t} \cdot\left((1-\mu) \frac{\partial v}{\partial x}-\frac{\partial v}{\partial y}\right)(Z(t)) d \mathcal{M}_{c}(t)\right] \\
& +\mathbb{E}^{x, y}\left[\sum_{0 \leq t_{k} \leq T_{R}} e^{-\delta t_{k}} \Delta_{\mathcal{L}, \mathcal{M}} v\left(Z\left(t_{k}\right)\right)\right]
\end{aligned}
$$

Note that by the mean value theorem we have

$$
\begin{aligned}
\Delta_{\mathcal{L}, \mathcal{M}} v\left(Z\left(t_{k}\right)\right) & =v\left(Z\left(t_{k}\right)\right)-v\left(Z\left(t_{k}^{-}\right)\right) \\
& =\frac{\partial v}{\partial x}\left(\hat{Z}_{(k)}\right) \Delta_{\mathcal{L}, \mathcal{M}} X\left(t_{k}\right)+\frac{\partial v}{\partial y}\left(\hat{Z}_{(k)}\right) \Delta_{\mathcal{L}, \mathcal{M}} Y\left(t_{k}\right) \\
& =\left(-(1+\lambda) \frac{\partial v}{\partial x}+\frac{\partial v}{\partial y}\right)\left(\hat{Z}_{(k)}\right) \Delta \mathcal{L}\left(t_{k}\right)+\left((1+\mu) \frac{\partial v}{\partial x}+\frac{\partial v}{\partial y}\right)\left(\hat{Z}_{(k)}\right) \Delta \mathcal{M}\left(t_{k}\right),
\end{aligned}
$$

where $\hat{Z}_{(k)}$ is some point on the line segment between $Z\left(t_{k}^{-}\right)$and $Z\left(t_{k}\right)$. Hence if (2.4), (2.5) and (2.6) hold then by (2.18) and (2.19) we get

$$
v(x, y) \geq \limsup _{R \rightarrow \infty} \mathbb{E}^{x, y}\left[\int_{0}^{T_{R}} e^{-\delta t} \frac{1}{\gamma} c^{\gamma}(t) d t+e^{-\delta T_{R}} v\left(Z\left(T_{R}\right)\right)\right] \geq J^{c, \mathcal{L}, \mathcal{M}}(x, y) .
$$

Since this holds for all admissible $(c, \mathcal{L}, \mathcal{M})$ we conclude that $(2.7)$ holds.

To prove b) we apply the above argument to the control $(\hat{c}, \hat{\mathcal{L}}, \hat{\mathcal{M}})$. Then by $(2.11)-(2.14)$ we get equality in the first part of (2.19), so that

$$
v(x, y)=\mathbb{E}^{x, y}\left[\int_{0}^{T_{R}} e^{-\delta t} \frac{1}{\gamma} \hat{c}^{\gamma}(t) d t+e^{-\delta T_{R}} v\left(\hat{Z}\left(T_{R}\right)\right)\right] .
$$

By (2.15) this gives

$$
v(x, y)=\mathbb{E}^{x, y}\left[\int_{0}^{\infty} e^{-\delta t} \frac{1}{\gamma} \hat{c}^{\gamma}(t) d t\right] \leq V(x, y) .
$$

Combined with (2.9) this gives the result.

Remark 2.3. Suppose the domain $D$ defined by (2.9) is known and has a smooth boundary (e.g. Lipschitz). Then one can regard (2.8) as a Neumann boundary value problem in $D$. In Section 4 we will discuss the relation between such problems and reflections/local time of diffusions in our jump diffusion case. Such a relation makes it possible to identify $(\hat{\mathcal{L}}(t), \hat{\mathcal{M}}(t))$ as the local time at $\partial D$ of the process $(\tilde{X}(t), \tilde{Y}(t))=\left(\tilde{X}^{\hat{c}}(t), \tilde{Y}(t)\right)$ obtained by reflecting $\left(X^{\hat{c}}(t), Y(t)\right)$ at $\partial D$ in the directions indicated by (2.11) and (2.12). 
Remark 2.4. Theorem 2.2 shows that it is natural to associate the integro-variational inequality

$$
\max \left\{L v(x, y), \sup _{c \geq 0}\left\{A^{c} v(x, y)+\frac{1}{\gamma} c^{\gamma}\right\}, M v(x, y)\right\}=0
$$

to the value function $V$ defined in (1.12). However, a priori we do not know if $V$ is smooth enough for $(2.21)$ to make sense in the usual way. Nevertheless, in the next section we will prove that $V$ solves an equation related to (2.21) in the weak sense of viscosity.

Before we do this we establish some other useful properties of $V$.

Lemma 2.5. a) Suppose (1.13) holds. Let $K_{0}$ be as in (1.15). Then

$$
V(x, y) \leq K_{0}(x+y)^{\gamma} \text { for all }(x, y) \in U
$$

b) Let $b$ be a constant such that

$$
1-\mu \leq b \leq 1+\lambda
$$

Suppose

$$
\delta>\gamma \alpha
$$

Then there exists $K<\infty$ such that

$$
V(x, y) \leq K(x+b y)^{\gamma} \text { for all }(x, y) \in U .
$$

Proof. Let $b$ be as in (2.23) and choose $K>0$. We will apply Theorem 2.2a) to the function

$$
v(x, y):=K(x+b y)^{\gamma} .
$$

First note that by $(2.23)$ we have

$$
L v=-(1+\lambda) \frac{\partial v}{\partial x}+\frac{\partial v}{\partial y}=(-(1+\lambda)+b) \gamma K(x+b y)^{\gamma-1} \leq 0
$$

and

$$
M v=(1-\mu) \frac{\partial v}{\partial x}-\frac{\partial v}{\partial y}=((1-\mu)-b) \gamma K(x+b y)^{\gamma-1} \leq 0
$$

so clearly (2.4) and (2.5) hold.

To verify $(2.6)$ we first note that

$$
\begin{aligned}
& \int_{-1}^{\infty}\left[v(x, y+y \eta)-v(x, y)-\frac{\partial v}{\partial y}(x, \eta) y \eta\right] d q(\eta) \\
= & K \int_{-1}^{\infty}\left[(x+b y+b y \eta)^{\gamma}-(x+b y)^{\gamma}-\gamma(x+b y)^{\gamma-1} b y \eta\right] d q(\eta) \\
= & K \gamma \int_{-1}^{\infty}\left[(x+b y+\theta b y \eta)^{\gamma-1}-(x+b y)^{\gamma-1}\right] b y \eta d q(\eta)
\end{aligned}
$$


for some $\theta \in(0,1)$ by the mean value theorem. This integrand is negative if $\eta>0$ (since $\gamma<1$ ) and also if $\eta<0$. Thus this integral is always nonpositive. Hence, for all $c \geq 0$ we have

$$
A^{c} v(x, y)+\frac{1}{\gamma} c^{\gamma} \leq-\delta v(x, y)+(r x-c) \frac{\partial v}{\partial x}+\alpha y \frac{\partial v}{\partial y}+\frac{1}{2} \sigma^{2} y^{2} \frac{\partial^{2} v}{\partial y^{2}}+\frac{1}{\gamma} c^{\gamma} .
$$

For $b=1$ and $K=K_{0}$ the last expression is nonpositive, for all $c \geq 0$, because we know that $v_{0}(x, y):=K_{0}(x+y)^{\gamma}$ solves the Hamilton-Jacobi-Bellman equation for the Merton problem $(q=$ $\lambda=\mu=0)$. We conclude that $v_{0}$ satisfies all the conditions of Theorem 2.2a) and a) follows.

To prove b) it suffices to verify that with $v$ as in (2.26) there exists $K<\infty$ such that

$$
H(c, x, y):=-\delta v+(r x-c) \frac{\partial v}{\partial x}+\alpha y \frac{\partial u}{\partial y}+\frac{1}{2} \sigma^{2} y^{2} \frac{\partial^{2} v}{\partial y^{2}}+\frac{1}{\gamma} c^{\gamma} \leq 0 .
$$

Since $c \mapsto H(c, x, y)$ is maximal when

$$
c=c^{*}=\left(\frac{\partial v}{\partial x}\right)^{\frac{1}{\gamma-1}}
$$

it suffices to obtain

$$
H_{0}(x, y):=H\left(c^{*}, x, y\right)=-\delta v+r x \frac{\partial v}{\partial x}+\alpha y \frac{\partial v}{\partial y}+\frac{1}{2} \sigma^{2} y^{2} \frac{\partial^{2} v}{\partial y^{2}}+\frac{1-\gamma}{\gamma}(K \gamma)^{\frac{\gamma}{\gamma-1}}(x+b y)^{\gamma} \leq 0
$$

which holds if

$$
\left[\frac{1-\gamma}{\gamma}(K \gamma)^{\frac{\gamma}{\gamma-1}}-\delta K+K \gamma \alpha\right](x+b y)^{2} \leq \frac{1}{2} \sigma^{2} K \gamma(1-\gamma) b^{2} y^{2}
$$

for all $x, y$. This holds if and only if the coefficient of $(x+b y)^{2}$ is nonpositive, i.e., if and only if

$$
\delta>\gamma \alpha+(\gamma K)^{\frac{1}{\gamma-1}}
$$

If (2.24) holds, then there exists $K$ such that (2.29) holds and therefore (2.6) holds for $v$ given by (2.26). This completes the proof of $b$ ).

Lemma 2.6. $V(x, y)$ is continuous on $\mathcal{S}$.

Proof. The continuity of $V$ on $\mathcal{S}^{0}$, the interior of $\mathcal{S}$, follows from the fact that $V$ is concave. (See e.g. $[\mathrm{R}])$. If $(\bar{x}, \bar{y}) \in \partial \mathcal{S}$ then the only admissible control is to take $\left(X_{\bar{x}}(t), Y_{\bar{y}}(t)\right)$ to the origin immediately, because otherwise diffusion will bring the process out of $\mathcal{S}$, almost surely. Hence $V(\bar{x}, \bar{y})=0$ for $(\bar{x}, \bar{y}) \in \partial \mathcal{S}$. So it remains to prove that

$$
\lim _{(x, y) \rightarrow(\bar{x}, \bar{y})} V(x, y)=0 \text { for all }(\bar{x}, \bar{y}) \in \partial \mathcal{S} .
$$

This follows from Lemma $2.5 \mathrm{~b}$ ) by choosing $b=1+\lambda$ and $b=1-\mu$.

Suppose the system is in state $z=(x, y)$ and we decide to make an admissible transaction $\ell=$ $\Delta \mathcal{L} \geq 0$ and $m=\Delta \mathcal{M} \geq 0$. Then the state jumps to $z^{\prime}=\left(x^{\prime}, y^{\prime}\right)$, where (see Figure 2)

$$
\begin{cases}x^{\prime} & =x-(1+\lambda) \ell+(1-\mu) m \\ y^{\prime} & =y+\ell-m\end{cases}
$$




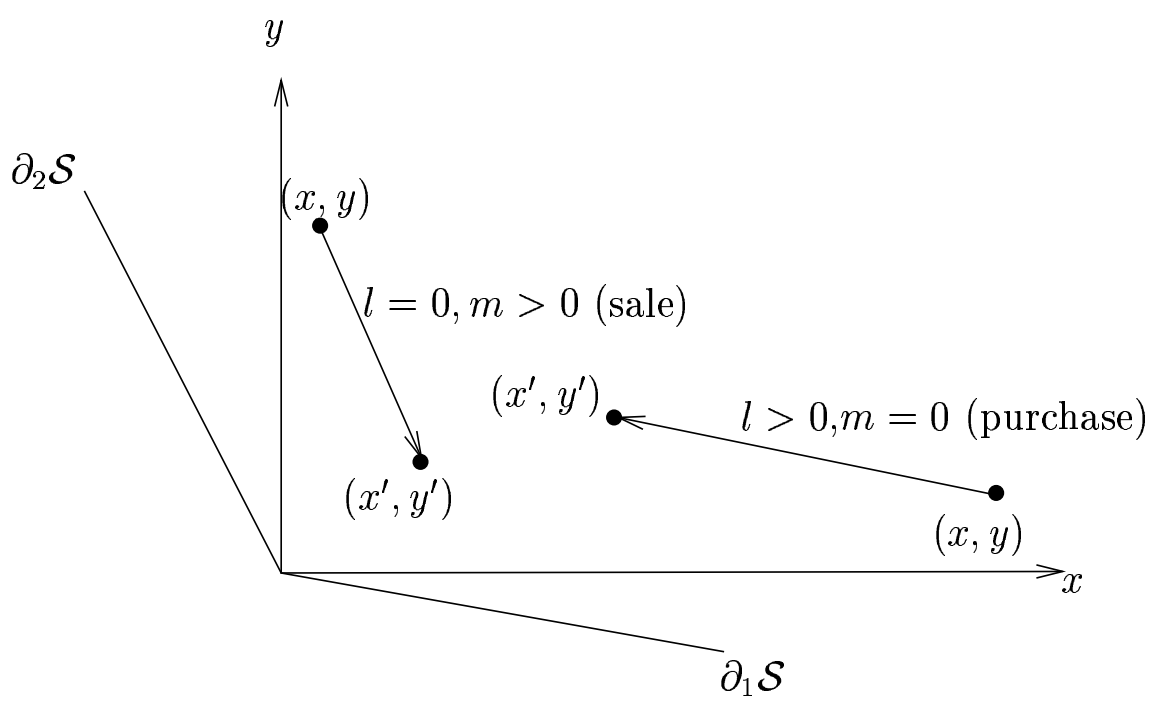

Figure 2: Transaction policies

Suppose we, when starting from $(x, y) \in \mathcal{S}$, make an immediate transaction which brings us to the state $\left(x^{\prime}, y^{\prime}\right) \in \mathcal{S}$ and from then on perform optimally from $\left(x^{\prime}, y^{\prime}\right)$. Then we get a performance which is at most as good as the best possible when starting from $(x, y)$.

Therefore we have

\section{Lemma 2.7.}

$$
V(x, y) \geq V\left(x^{\prime}, y^{\prime}\right) \text { for all }(x, y) \in \mathcal{S}, \quad \ell \geq 0, m \geq 0 .
$$

Now fix $(x, y) \in \mathcal{S}^{0}$ and consider the function

$$
g(\ell)=g_{x, y}(\ell)=V(x-(1+\lambda) \ell, y+\ell) ; \quad 0 \leq \ell \leq \bar{\ell}
$$

where

$$
\bar{\ell}=\sup \{\ell>0 ;(x-(1+\lambda) \ell, y+\ell) \in \mathcal{S}\} .
$$

It follows from Lemma 2.7 that $g$ is a decreasing function. Hence $g^{\prime}(\ell) \leq 0$ for a.a. $\ell \in(0, \bar{\ell})$. Now $g^{\prime}(\ell)$ is just the directional derivative of $V$ in the direction $(-(1+\lambda), 1)$ and since $\frac{\partial V}{\partial x}$ exists for a.a. $x$ for each $y$ (by Lemma 2.1a)) we conclude that

$$
g^{\prime}(\ell)=-(1+\lambda) \frac{\partial V}{\partial x}+\frac{\partial V}{\partial y} \text { for a.a. } x, y, \ell
$$

This gives part a) of the following result:

Lemma 2.8. a) $-(1+\lambda) \frac{\partial V}{\partial x}+\frac{\partial V}{\partial y} \leq 0$ for a.a. $(x, y) \in \mathcal{S}$,

b) $(1-\mu) \frac{\partial V}{\partial x}-\frac{\partial V}{\partial y} \leq 0$ for a.a. $(x, y) \in \mathcal{S}$. 
Part b) of this Lemma is proved similarly to part a) by replacing $g$ by the function

$$
h(m):=V(x+(1-\mu) m, y-m) ; \quad 0 \leq m \leq \bar{m},
$$

where

$$
\bar{m}=\sup \{m>0 ;(x+(1-\mu) m, y-m) \in \mathcal{S}\} .
$$

Note that $g$ and $h$ are concave functions, because they are just the restriction of the concave function $V$ to straight lines.

For an arbitrary continuous function $v: \mathcal{S} \mapsto \mathbb{R}$ we now define $\hat{L} v$ and $\hat{M} v$ by

$$
\begin{gathered}
\hat{L} v(x, y)=\left\{\begin{array}{cl}
-1 & \text { if } v(x-(1+\lambda) \ell, y+\ell)<v(x, y) \text { for all } \ell \in(0, \bar{\ell}) \\
0 & \text { if } v(x-(1+\lambda) \ell, y+\ell)=v(x, y) \text { for some } \ell \in(0, \bar{\ell}) \\
+1 & \text { if } v(x-(1+\lambda) \ell, y+\ell)>v(x, y) \text { for all } \ell \in(0, \bar{\ell})
\end{array}\right. \\
\hat{M} v(x, y)=\left\{\begin{array}{cl}
-1 & \text { if } v(x+(1-\mu) m, y-m)<v(x, y) \text { for all } m \in(0, \bar{m}) \\
0 & \text { if } v(x+(1-\mu) m, y-m)=v(x, y) \text { for some } m \in(0, \bar{m}) \\
+1 & \text { if } v(x+(1-\mu) m, y-m)>v(x, y) \text { for all } m \in(0, \bar{m})
\end{array}\right.
\end{gathered}
$$

Then we have seen above that

$$
\hat{L} V(x, y) \leq 0 \text { and } \hat{M} V(x, y) \leq 0 \text { for all }(x, y) \in \mathcal{S}^{0} .
$$

Moreover, if $\hat{L} V\left(x_{0}, y_{0}\right)<0$ then we must have $g^{\prime}(\ell)<0$ for arbitrary small $\ell>0$ and hence for almost all $\ell>0$ by concavity. A similar argument works for $h(m)$. This proves:

Lemma 2.9. a) If $\hat{L} V\left(x_{0}, y_{0}\right)<0$, then $-(1+\lambda) \frac{\partial V}{\partial x}+\frac{\partial V}{\partial y}<0$

at almost all points $(x, y)=\left(x_{0}-(1+\lambda) \ell, y_{0}+\ell\right), \quad 0<\ell<\bar{\ell}$.

b) If $\hat{M} V\left(x_{0}, y_{0}\right)<0$, then $(1-\mu) \frac{\partial V}{\partial x}-\frac{\partial V}{\partial y}<0$

at almost all points $(x, y)=\left(x_{0}+(1-\mu) m, y_{0}-m\right), \quad 0<m<\bar{m}$.

Theorem 2.10. There exist real numbers $\theta_{1}^{*}, \theta_{2}^{*}$ with $\theta_{1} \leq \theta_{1}^{*}<\theta_{2}^{*} \leq \theta_{2}$ such that if we define

$$
B=\left\{(x, y)=R e^{i \theta} \in U ; \quad R \geq 0 ; \quad \theta \leq \theta_{1}^{*}\right\} \quad \text { (the buy region) }
$$

and

$$
S=\left\{(x, y)=R e^{i \theta} \in U ; \quad R \geq 0 ; \quad \theta \geq \theta_{2}^{*}\right\} \quad \text { (the sell region) }
$$

then

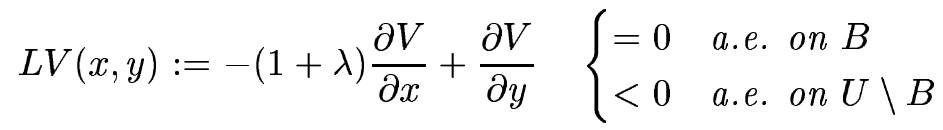

and

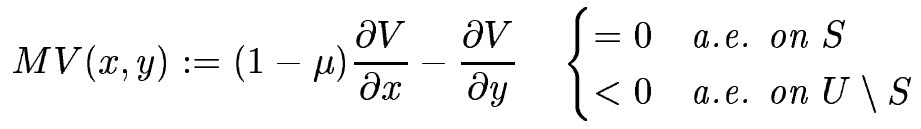




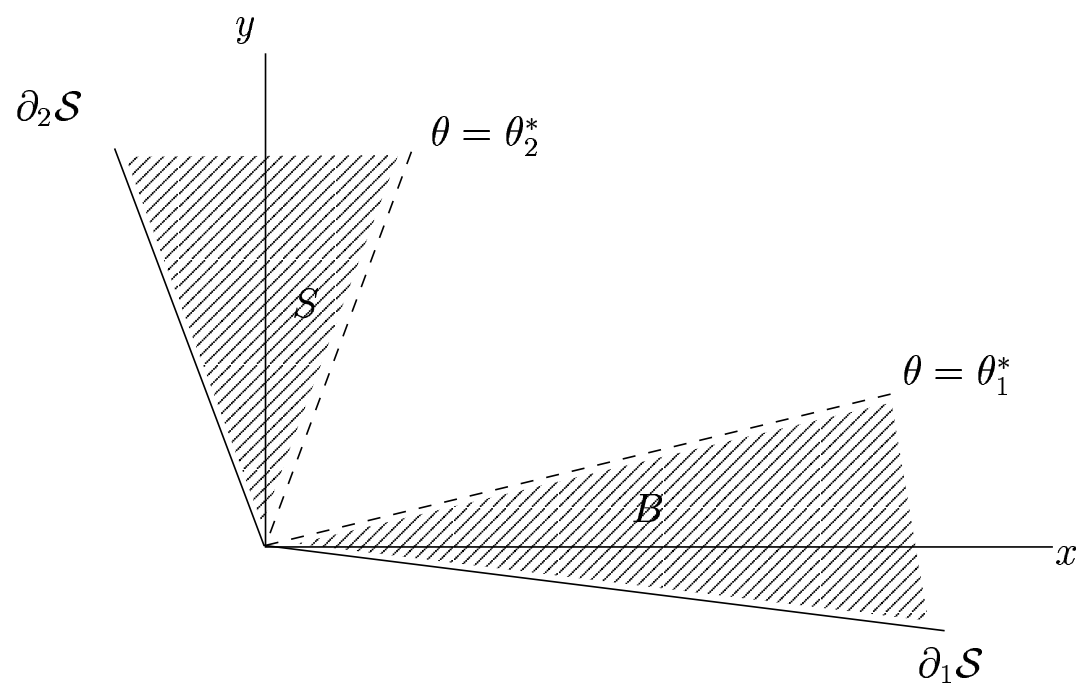

Figure 3: The buy and sell regions

Proof. Following [AMS] we introduce the new variables

$$
\rho=x+(1-\mu) y \quad(\text { net wealth })
$$

and

$$
\beta=\frac{(1-\mu) y}{\rho} \quad \text { (fraction of net wealth invested in stock). }
$$

Then by homogeneity we can write

$$
V(x, y)=V\left(\rho(1-\beta), \rho \frac{\beta}{1-\mu}\right)=\rho^{\gamma} V\left(1-\beta, \frac{\beta}{1-\mu}\right)=\rho^{\gamma} G(\beta),
$$

where

$$
G(\beta)=V\left(1-\beta, \frac{\beta}{1-\mu}\right), \quad \beta>-\frac{1-\mu}{\lambda+\mu} .
$$

Note that $G(\beta)$ is concave, because it is just the restriction of a concave function to a straight line. Moreover,

$$
G^{\prime}(\beta)=(-1) \frac{\partial V}{\partial x}+\frac{1}{1-\mu} \frac{\partial V}{\partial y}=-\frac{1}{1-\mu}\left[(1-\mu) \frac{\partial V}{\partial x}-\frac{\partial V}{\partial y}\right]
$$

Suppose there exists $\beta^{*}>-\frac{1-\mu}{\lambda+\mu}$ such that $G^{\prime}\left(\beta^{*}\right) \leq 0$. Then by concavity we must have $G^{\prime}(\beta) \leq 0$ for a.a. $\beta \geq \beta^{*}$. On the other hand, by Lemma $2.8 \mathrm{~b}$ ) we also have $G^{\prime}(\beta) \geq 0$ for a.a. $\beta$. We conclude that $G^{\prime}(\beta)=0$ for a.a. $\beta \geq \beta^{*}$. Therefore, if we define

$$
\beta_{2}^{*}=\inf \left\{\beta^{*} ; G^{\prime}\left(\beta^{*}\right) \leq 0\right\}
$$


we have

$$
\begin{aligned}
& G^{\prime}(\beta)>0 \text { for a.a. } \beta<\beta_{2}^{*} \text { and } \\
& G^{\prime}(\beta)=0 \text { for a.a. } \beta>\beta_{2}^{*} .
\end{aligned}
$$

Since

$$
\beta>\beta_{2}^{*} \Leftrightarrow \theta>\theta_{2}^{*}, \quad \text { where } \tan \theta_{2}^{*}=\frac{\beta_{2}^{*}}{\left(1-\beta_{2}^{*}\right)(1-\mu)}
$$

we conclude that (2.37) holds with

$$
\tan \theta_{2}^{*}=\frac{\beta_{2}^{*}}{\left(1-\beta_{2}^{*}\right)(1-\mu)}
$$

Similarly, by using the coordinates

$$
\hat{\rho}=x+(1+\lambda) y, \quad \hat{\beta}=\frac{(1+\lambda) y}{\rho}
$$

we deduce that (2.36) holds.

\section{Viscosity solutions}

Let $L, M$ and $A^{c}$ be as in Theorem 2.2, $\hat{L}, \hat{M}$ as in (2.32), (2.33) and define

$$
\begin{aligned}
\tilde{L} u(x, y) & =\max (L u(x, y), \hat{L} u(x, y)) \\
\tilde{M} u(x, y) & =\max (M u(x, y), \hat{M} u(x, y)) .
\end{aligned}
$$

We consider the following integro-variational inequality associated to (2.21):

$$
\max \left\{\tilde{L} u(x, y), \sup _{c \geq 0}\left\{A^{c} u(x, y)+\frac{1}{\gamma} c^{\gamma}\right\}, \tilde{M} u(x, y)\right\}=0 \text { in } U .
$$

We define viscosity solutions of (3.3) as follows:

Definition 3.1. a) A function $u \in C(U)$ is a viscosity subsolution in $U$ of (3.3) if, for all functions $w \in C^{2}(U)$ and all $z_{0}=\left(x_{0}, y_{0}\right) \in U$ such that $w \geq u$ on $U$ and $w\left(z_{0}\right)=u\left(z_{0}\right)$ we have

$$
\max \left\{\tilde{L} w\left(z_{0}\right), \sup _{c \geq 0}\left\{A^{c} w\left(z_{0}\right)+\frac{1}{\gamma} c^{\gamma}\right\}, \tilde{M} w\left(z_{0}\right)\right\} \geq 0 .
$$

b) A function $u \in C(U)$ is a viscosity supersolution of (3.3) if, for all functions $w \in C^{2}(U)$ and all $z_{0}=\left(x_{0}, y_{0}\right) \in U$ such that $w \leq u$ on $U$ and $w\left(z_{0}\right)=u\left(z_{0}\right)$ we have

$$
\max \left\{\tilde{L} w\left(z_{0}\right), \sup _{c \geq 0}\left\{A^{c} w\left(z_{0}\right)+\frac{1}{\gamma} c^{\gamma}\right\}, \tilde{M} w\left(z_{0}\right)\right\} \leq 0 .
$$

c) A continuous function $u$ on $U$ is called a viscosity solution of (3.3) if it is both a viscosity subsolution and a viscosity supersolution. 
Theorem 3.2. Let $V$ be the value function for problem (1.12). Then $V$ is a viscosity solution of (3.3) on $U$.

Proof. We know that $V$ is continuous.

(i): $V$ is a subsolution

Choose $w \in C^{2}(U)$ and $z_{0} \in U$ such that $w \geq V$ on $U$ on $w\left(z_{0}\right)=V\left(z_{0}\right)$. By the dynamic programming principle we have

$$
V\left(z_{0}\right)=\sup _{w \in \mathcal{A}\left(z_{0}\right)} \mathbb{E}^{z_{0}}\left[\int_{0}^{\tau} e^{-\delta t} \frac{c^{\gamma}(t)}{\gamma} d t+e^{-\delta \tau} V(Z(\tau))\right]
$$

for all stopping times $\tau$. Let $\epsilon>0$ and choose an $\epsilon$-optimal admissible control $\tilde{w}=(\tilde{c}, \tilde{\mathcal{L}}, \tilde{\mathcal{M}})$, so that for all stopping times $\tau$ :

$$
V\left(z_{0}\right) \leq \mathbb{E}^{z_{0}}\left[\int_{0}^{\tau} e^{-\delta t} \frac{\tilde{c}^{\gamma}(t)}{\gamma} d t+e^{-\delta \tau} V(\tilde{Z}(\tau))\right]+\epsilon
$$

where $\tilde{Z}=Z^{\tilde{w}}$. On the other hand, by the Ito formula we have (see (2.18))

$$
\begin{aligned}
\mathbb{E}^{z_{0}}\left[e^{-\delta \tau} w(Z(\tau))\right]= & w\left(z_{0}\right)+\mathbb{E}^{z_{0}}\left[\int_{0}^{\tau} e^{-\delta t} A^{c} w(Z(t)) d t\right] \\
& +\mathbb{E}^{z_{0}}\left[\int_{0}^{\tau} e^{-\delta t} \cdot\left(-(1+\lambda) \frac{\partial w}{\partial x}+\frac{\partial w}{\partial y}\right)(Z(t)) d \mathcal{L}_{c}(t)\right] \\
& +\mathbb{E}^{z_{0}}\left[\int_{0}^{\tau} e^{-\delta t} \cdot\left((1-\mu) \frac{\partial w}{\partial x}-\frac{\partial w}{\partial y}\right)(Z(t)) d \mathcal{M}_{c}(t)\right] \\
& +\mathbb{E}^{z_{0}}\left[\sum_{0 \leq t_{k} \leq \tau} e^{-\delta t_{k}} \Delta_{\mathcal{L}, \mathcal{M}} w\left(Z\left(t_{k}\right)\right)\right]
\end{aligned}
$$

for all admissible controls $w=(c, \mathcal{L}, \mathcal{M})$ and all stopping times $\tau$ satisfying

$$
\tau \leq \tau_{M}:=M \wedge \inf \{t>0 ;|Z(t)| \geq M \text { or } Z(t) \in \partial \mathcal{S}\}
$$

for some $M<\infty$. Combining this with (3.7) we get, with $\tilde{Z}=Z^{\tilde{w}}$,

$$
\begin{aligned}
0 \leq \epsilon & +\mathbb{E}^{z_{0}}\left[\int_{0}^{\tau} e^{-\delta t}\left(A^{\tilde{c}} w(\tilde{Z}(t))+\frac{(\tilde{c})^{\gamma}(t)}{\gamma}\right) d t\right] \\
& +\mathbb{E}^{z_{0}}\left[\int_{0}^{\tau} e^{-\delta t} \cdot\left(-(1+\lambda) \frac{\partial w}{\partial x}+\frac{\partial w}{\partial y}\right)(\tilde{Z}(t)) d \tilde{\mathcal{L}}_{c}(t)\right] \\
& +\mathbb{E}^{z_{0}}\left[\int_{0}^{\tau} e^{-\delta t} \cdot\left((1-\mu) \frac{\partial w}{\partial x}-\frac{\partial w}{\partial y}\right)(\tilde{Z}(t)) d \tilde{\mathcal{M}}_{c}(t)\right] \\
& +\mathbb{E}^{z_{0}}\left[\sum_{0 \leq t_{k} \leq \tau} e^{-\delta t_{k}} \Delta_{\tilde{\mathcal{L}}, \tilde{\mathcal{M}}} w\left(\tilde{Z}\left(t_{k}\right)\right)\right] .
\end{aligned}
$$

Let $\tilde{t}_{1}$ be the first jumping time of $(\tilde{\mathcal{L}}(t), \tilde{\mathcal{M}}(t))$. Note that since $\tilde{t}_{1}$ is an $\mathcal{F}_{t}$-stopping time we know that $\left\{\omega ; \tilde{t}_{1}(\omega)=0\right\}$ is $\mathcal{F}_{0}$-measurable and hence this event has probability either 0 or 1 . So we have either $\tilde{t}_{1}(\omega)=0$ a.s. or $\left.\tilde{t}_{1}(\omega)\right)>0$ a.s. Let us first assume that $\tilde{t}_{1}(\omega)>0$ a.s. Choose

$$
0<\tau \leq \tilde{t}_{1} \wedge \tau_{M}
$$


Suppose $\tilde{L} w\left(z_{0}\right)<0$ and $\tilde{M} w\left(z_{0}\right)<0$. Then

$$
\begin{aligned}
\left(-(1+\lambda) \frac{\partial w}{\partial x}+\frac{\partial w}{\partial y}\right)\left(z_{0}\right) & <0 \\
\left((1-\mu) \frac{\partial w}{\partial x}-\frac{\partial w}{\partial y}\right)\left(z_{0}\right) & <0
\end{aligned}
$$

Hence by choosing $\tau$ to be smaller than the first exit time from a small enough neighbourhood of $z_{0}$ we get from (3.9) that

$$
\mathbb{E}^{z_{0}}\left[\int_{0}^{\tau} e^{-\delta t}\left(A^{\tilde{c}} w(\tilde{Z}(t))+\frac{(\tilde{c})^{\gamma}(t)}{\gamma}\right) d t\right] \geq-\epsilon
$$

Suppose

$$
R w\left(z_{0}\right):=\sup _{c \geq 0}\left\{A^{c} w\left(z_{0}\right)+\frac{c^{\gamma}}{\gamma}\right\}=2 \beta<0
$$

Then necessarily $\frac{\partial w}{\partial x}\left(z_{0}\right)>0$ and hence by continuity $\frac{\partial w}{\partial x}>0$ on some neighbourhood $A$ of $z_{0}$. But then

$$
R w\left(z_{0}\right)=\frac{\partial w}{\partial s}+(r x-\hat{c}) \frac{\partial w}{\partial x}+\alpha y \frac{\partial w}{\partial y}+\frac{1}{2} \sigma^{2} \frac{\partial^{2} w}{\partial y^{2}}+\frac{\hat{c}^{\gamma}}{\gamma}
$$

with $\hat{c}=\hat{c}(z)=\left(\frac{\partial w}{\partial x}\right)^{\frac{1}{\gamma-1}}$ on $A$. It follows that $R w$ is continuous on $A$. Choosing $\tau$ small enough, we have $e^{-\delta t} R<\beta$ for $0<t<\tau$ and from (3.10) we must have $0 \leq \beta \mathbb{E}^{z_{0}}[\tau]+\epsilon$. Now let $\epsilon \rightarrow 0$ to obtain a contradiction. We conclude that (3.4) holds if $\tilde{t}_{1}>0$. Next, if $\tilde{t}_{1}=0$ a.s. then we choose $\tau=0$ in (3.9) and get

$$
\mathbb{E}^{z_{0}}\left[\Delta_{\tilde{\mathcal{L}, \mathcal{M}}} w(Z(0))\right] \geq-\epsilon
$$

This implies that either $\tilde{L} w\left(z_{0}\right) \geq 0$ or $\tilde{M} w\left(z_{0}\right) \geq 0$, so (3.4) holds in this case also. Hence $V$ is a subsolution.

(ii): $V$ is a supersolution.

Choose $w \in C^{2}(U)$ and $z_{0} \in U$ such that $w \leq V$ on $U$ and $w\left(z_{0}\right)=V\left(z_{0}\right)$. Then by combining (3.6) and (3.8) we get, for all admissible controls $w=(c, \mathcal{L}, \mathcal{M})$ and all $\tau \leq \tau_{M}$,

$$
\begin{aligned}
0 \geq & \mathbb{E}^{z_{0}}\left[\int_{0}^{\tau} e^{-\delta t}\left(A^{c} w(Z(t))+\frac{c^{\gamma}(t)}{\gamma}\right) d t\right] \\
& +\mathbb{E}^{z_{0}}\left[\int_{0}^{\tau} e^{-\delta t}\left(-(1+\lambda) \frac{\partial w}{\partial x}+\frac{\partial w}{\partial y}\right)(Z(t)) d \mathcal{L}_{c}(t)\right] \\
& +\mathbb{E}^{z_{0}}\left[\int_{0}^{\tau} e^{-\delta t}\left((1-\mu) \frac{\partial w}{\partial x}-\frac{\partial w}{\partial y}\right)(Z(t)) d \mathcal{M}_{c}(t)\right] \\
& +\mathbb{E}^{z_{0}}\left[\sum_{0 \leq t_{k} \leq \tau} e^{-\delta t_{k}} \Delta_{\mathcal{L}, \mathcal{M}} w\left(Z\left(t_{k}\right)\right)\right]
\end{aligned}
$$

Now choose $\mathcal{M}=0$ and $\mathcal{L}(t)$ to make an immediate jump to $\ell>0$ at time $t=0$. Then by (3.13) we get

$$
\mathbb{E}^{z_{0}}\left[\Delta_{\mathcal{L}, \mathcal{M}} w(Z(0))\right] \leq 0
$$


i.e.,

$$
w\left(x_{0}-(1+\lambda) \ell, y_{0}+\ell\right) \leq w\left(x_{0}, y_{0}\right) .
$$

This implies that $\tilde{L} w\left(x_{0}, y_{0}\right) \leq 0$. Similarly we obtain $\tilde{M} w\left(x_{0}, y_{0}\right) \leq 0$. Finally, by choosing $w=(c, 0,0)$ for $t \leq \tau$ with $c \geq 0$ constant (and $\tau$ so small that $w$ is admissible) we get by (3.13) that

$$
\mathbb{E}^{z_{0}}\left[\int_{0}^{\tau} e^{-\delta t}\left(A^{c} w(Z(t))+\frac{c^{\gamma}}{\gamma}\right) d t\right] \leq 0 .
$$

Dividing by $\mathbb{E}^{z_{0}}[\tau]$ and letting $\tau \rightarrow 0$ we get

$$
A^{c} w\left(z_{0}\right)+\frac{c^{\gamma}}{\gamma} \leq 0 .
$$

We conclude that (3.5) holds. So $V$ is a viscosity supersolution.

Corollary 3.3. Suppose $V \in C^{2}(U)$. Then

$$
\max \left\{L V(x, y), \sup _{c \geq 0}\left\{A^{c} V(x, y)+\frac{1}{\gamma} c^{\gamma}\right\}, M V(x, y)\right\}=0 \text { on } U .
$$

Moreover, with B,S as in Theorem 2.10 we have

$$
\begin{aligned}
L V(x, y)=0 & \Longleftrightarrow(x, y) \in B \\
M V(x, y)=0 & \Longleftrightarrow(x, y) \in S \\
\sup _{c \geq 0}\left\{A^{c} V(x, y)+\frac{1}{\gamma} c^{\gamma}\right\}=0 & \Longleftrightarrow(x, y) \in U \backslash\left(B^{0} \cup S^{0}\right) .
\end{aligned}
$$

Proof. Since $V$ satisfies (3.3) in viscosity sense and $V \in C^{2}(U)$ we know that $V$ also satisfies (3.3) in the ordinary sense. This immediately implies that the left hand side of $(3.15)$ is $\leq 0$. On the other hand, if $(x, y) \in U$ is such that

$$
L V(x, y)<0 \text { and } M V(x, y)<0
$$

then clearly $\hat{L} V(x, y)=-1$ and $\hat{M} V(x, y)=-1$, so $\tilde{L} V(x, y)<0$ and $\tilde{M} V(x, y)<0$. Therefore by $(3.3)$

$$
\sup _{c \geq 0}\left\{A^{c} V(x, y)+\frac{1}{\gamma} c^{\gamma}\right\}=0 .
$$

This proves that (3.15) holds. Then (3.16) - (3.18) follow from Theorem 2.10.

\section{Reflected jump diffusions and identification of the optimal port- folio}

In this section we identify the optimal portfolio with the local time of a reflected jump diffusion. We start with the following result, which is an adaptation of Theorem 15 in [CEM]. 
Theorem 4.1. Let $c(t) \geq 0$ be a given adapted process. Fix $\hat{\theta}_{1}, \hat{\theta}_{2}$ such that

$$
\theta_{1} \leq \hat{\theta}_{1}<\hat{\theta}_{2} \leq \theta_{2}
$$

and define

$$
\begin{aligned}
D & =\left\{(x, y)=R e^{i \theta} ; \quad R \geq 0 ; \quad \hat{\theta}_{1}<\theta<\hat{\theta}_{2}\right\} \\
\partial_{k} D & =\left\{(x, y)=R e^{i \theta} ; \quad R \geq 0 ; \quad \theta=\hat{\theta}_{k}\right\}, \quad k=1,2 \\
B & =\left\{(x, y)=R e^{i \theta} \in \mathcal{S} ; \quad R \geq 0 ; \quad \theta \leq \hat{\theta}_{1}\right\} \\
S & =\left\{(x, y)=R e^{i \theta} \in \mathcal{S} ; \quad R \geq 0 ; \quad \hat{\theta}_{2} \leq \theta\right\} .
\end{aligned}
$$

Then there exist unique adapted processes $\tilde{X}(t), \tilde{Y}(t), \hat{\mathcal{L}}(t), \hat{\mathcal{M}}(t)$ satisfying the following Skorohod stochastic differential equation, given by the set of conditions $(4.5)-(4.8)$ :

$$
\begin{gathered}
d \tilde{X}(t)=(r \tilde{X}(t)-c(t)) d t-(1+\lambda) d \tilde{\mathcal{L}}(t)+(1-\mu) d \tilde{\mathcal{M}}(t) ; \quad X\left(0^{-}\right)=x \in \mathbb{R} \\
d \tilde{Y}(t)=\tilde{Y}\left(t^{-}\right)\left(\alpha d t+\sigma d W(t)+\int_{-1}^{\infty} \eta \tilde{N}(d t, d \eta)\right)+d \tilde{\mathcal{L}}(t)-d \tilde{\mathcal{M}}(t) ; \quad Y\left(0^{-}\right)=y \in \mathbb{R} \\
(\tilde{X}(t), \tilde{Y}(t)) \in \bar{D} \text { for all } t>0
\end{gathered}
$$

$\hat{\mathcal{L}}(t), \hat{\mathcal{M}}(t)$ are nondecreasing and their continuous parts, $\hat{\mathcal{L}}_{c}(t), \hat{\mathcal{M}}_{c}(t)$, increase only when $(\tilde{X}(t), \tilde{Y}(t)) \in \partial_{1} D, \quad(\tilde{X}(t), \tilde{Y}(t)) \in \partial_{2} D$, respectively

$$
\begin{aligned}
\Delta \hat{\mathcal{L}}(t)>0 & \text { if and only if }\left(X\left(t^{-}\right), Y\left(t^{-}\right)+\Delta_{N} Y(t)\right) \\
\Delta \hat{\mathcal{M}}(t)>0 & \in B^{0}, \\
\text { if and only if }\left(X\left(t^{-}\right), Y\left(t^{-}\right)+\Delta_{N} Y(t)\right) & \in S^{0},
\end{aligned}
$$

and if this is the case then

$$
\begin{aligned}
\Delta \hat{\mathcal{L}}(t) & =\min \left\{\ell>0 ;\left(X\left(t^{-}\right)-(1+\lambda) \ell, Y(t-)+\Delta_{N} Y(t)+\ell\right) \notin B^{0}\right\} \\
\Delta \hat{\mathcal{M}}(t) & =\min \left\{m>0 ;\left(X\left(t^{-}\right)+(1-\mu) m, Y\left(t^{-}\right)+\Delta_{N} Y(t)-m\right) \notin S^{0}\right\}
\end{aligned}
$$

with $\Delta_{N} Y(t)$ as in $(1.10)$.

Remark 4.2. The process $(\tilde{X}(t), \tilde{Y}(t))$ is called the reflection of the process $\left(X^{(c, 0,0)}(t), Y^{(c, 0,0)}(t)\right)$ in the directions $(-(1+\lambda), 1)$ and $((1-\mu),-1)$ at the two boundary curves $\partial_{1} D$ and $\partial_{2} D$ of $D$. Note that we only have $\Delta \hat{\mathcal{L}}(t)>0$ or $\Delta \hat{\mathcal{M}}(t)>0$ if either $t=0$ and $Z\left(0^{-}\right) \notin \bar{D}$ or if $Z(t)$ (by the jump in the random measure term) jumps out of $\bar{D}$. In these cases we either buy $(\Delta \hat{\mathcal{L}}>0)$ or sell $(\Delta \hat{\mathcal{M}}>0)$ immediately to bring $Z(t)$ to $\partial D$. See Figure 4 . The two processes $\hat{\mathcal{L}}(t), \hat{\mathcal{M}}(t)$ are called the corresponding local times of $(\tilde{X}(t), \tilde{Y}(t))$ at $\partial_{1} D, \partial_{2} D$. We see that $\hat{\mathcal{L}}(t), \hat{\mathcal{M}}(t)$ satisfy the equations (2.11) - (2.12).

Finally we use this to give an explicit description of the optimal control for problem (1.12), under some conditions: 


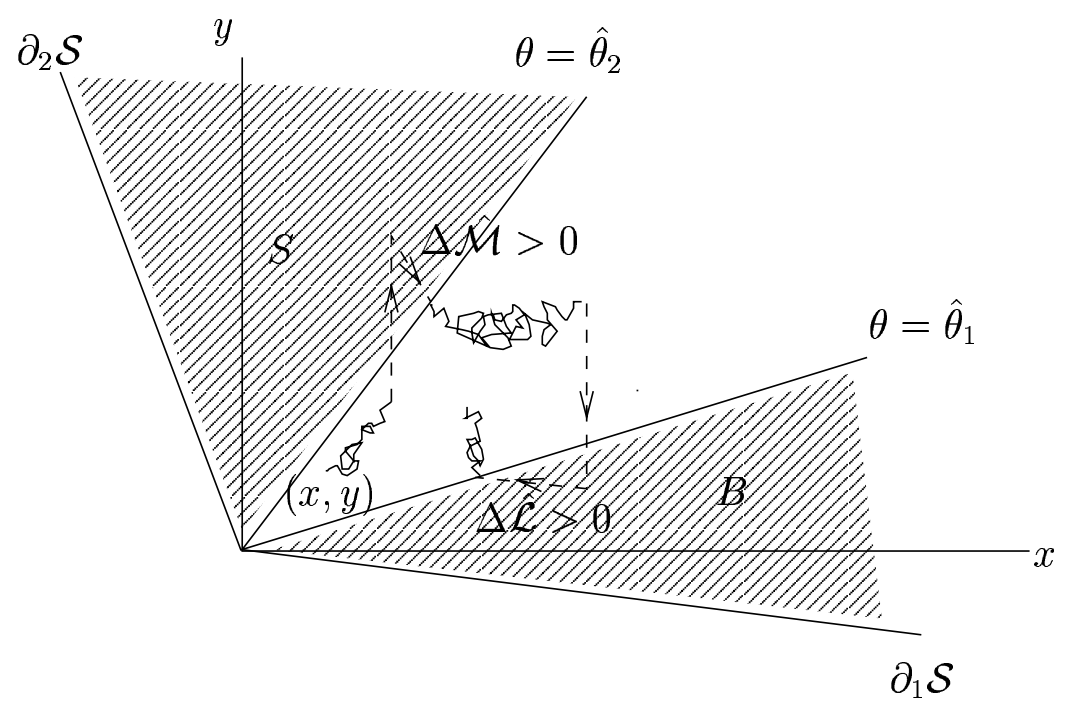

Figure 4: Optimal wealth process and corresponding local times investment policies

Theorem 4.3. Suppose $V \in C^{2}(U)$ and let $\theta_{1} \leq \theta_{1}^{*}<\theta_{2}^{*} \leq \theta_{2}$ be as in Theorem 2.10. Suppose that

$$
0 \leq \theta_{1}^{*}<\theta_{2}^{*} \leq \frac{\pi}{2}
$$

and that

$$
\delta>\gamma \alpha-\frac{1}{2} \sigma^{2} \gamma(1-\gamma)-\gamma\|q\|+\int_{-1}^{\infty}\left((1+\eta)^{\gamma}-1\right) d q(\eta)
$$

Define

$$
\left(X^{*}, Y^{*}, \mathcal{L}^{*}, \mathcal{M}^{*}\right)=(\tilde{X}, \tilde{Y}, \hat{\mathcal{L}}, \hat{\mathcal{M}})
$$

where $(\tilde{X}, \tilde{Y}, \hat{\mathcal{L}}, \hat{\mathcal{M}})$ is the solution of the Skorohod equation in Theorem 4.1 with

$$
\hat{\theta}_{1}=\theta_{1}^{*}, \quad \hat{\theta}_{2}=\theta_{2}^{*}
$$

Put

$$
c^{*}(x, y)=\left(\frac{\partial V}{\partial x}\right)^{\frac{1}{\gamma-1}} .
$$

Then $w^{*}=\left(c^{*}, \mathcal{L}^{*}, \mathcal{M}^{*}\right)$ is an optimal control for problem $(1.12)$ and $Z^{*}(t)=\left(X^{*}(t), Y^{*}(t)\right)$ is the corresponding optimal state process.

Proof. We apply Theorem 2.2b) with $v=V$. By Corollary 3.3, we know that

$$
\sup _{c \geq 0}\left\{A^{c} V(x, y)+\frac{1}{\gamma} c^{\gamma}\right\}=0
$$


for all $(x, y)=R e^{i \theta}$ with $\theta_{1}^{*}<\theta<\theta_{2}^{*}$. This implies that $\frac{\partial V}{\partial x}(x, y)>0$ and that the value of $c$ for which the supremum in (4.13) is attained is

$$
c=c^{*}(x, y)=\left(\frac{\partial V}{\partial x}\right)^{\frac{1}{\gamma-1}} .
$$

Hence, with $\hat{c}=c^{*}$ we have (2.8) satisfied. Next, with $\hat{\mathcal{L}}, \hat{\mathcal{M}}$ as in Theorem 4.1 we see from (4.7) that (2.11) and (2.12) holds, while (4.6) implies (2.14). Moreover, by (4.8) and (3.16) - (3.17) we know that $V$ does not change value under any of the jumps of $Z(t)$ casused by the jumps of $(\hat{\mathcal{L}}(t), \hat{\mathcal{M}}(t))$. Hence $(2.13)$ holds.

It remains to verify $(2.15)$. Since $V(x, y) \leq K_{0}(x+y)^{\gamma}$ (by Lemma 2.5a)) it suffices to prove that

$$
\lim _{R \rightarrow \infty} \mathbb{E}^{x, y}\left[e^{-\delta T_{R}}\left(X^{w^{*}}\left(T_{R}\right)+Y^{w^{*}}\left(T_{R}\right)\right)^{\gamma}\right]=0
$$

To this end, note that from the dynamics of $X$ and $Y$, combined with (4.9) we have, with $Z^{*}=Z^{w^{*}}$,

$$
d Z^{*}(t) \leq \alpha Z^{*}(t) d t+\sigma Z^{*}(t) d W(t)+Z^{*}\left(t^{-}\right) \int_{-1}^{\infty} \eta \tilde{N}(d t, d \eta)
$$

Hence (see the proof of Theorem 2.3 in [FØS] for details)

$$
Z^{*}(t) \leq Z(0) \exp \left\{\left(\alpha-\frac{1}{2} \sigma^{2}-\|q\|\right) t+\sigma W(t)+\int_{0}^{t} \int_{-1}^{\infty} \ln (1+\eta) N(d s, d \eta)\right\}
$$

and hence

$$
\begin{aligned}
& \mathbb{E}\left[e^{-\delta T_{R}}\left(Z^{*}\left(T_{R}\right)\right)^{\gamma}\right] \\
& \quad \leq Z^{\gamma}(0) \mathbb{E}\left[\exp \left\{\left(-\delta+\gamma \alpha-\frac{1}{2} \sigma^{2} \gamma(1-\gamma)-\gamma\|q\|+\int_{-1}^{\infty}\left((1+\eta)^{\gamma}-1\right) d q(\eta)\right) T_{R}\right\}\right]
\end{aligned}
$$

which goes to 0 as $R \rightarrow \infty$ because by (4.10) the coefficient of $T_{R}$ in the exponent is negative.

\section{For future research}

Economic intuition suggests the following properties:

- The continuation region tends to the first quadrant as $\lambda \rightarrow \infty$ and $\mu \rightarrow 1$. It is tempting to guess that the boundaries of the no transaction region tend monotonically to the axes. This agrees with the conjecture in [SS, Remark 11.3] in that when leverage is optimal in the Merton problem, then the presence of transaction cost will reduce the leverage. We expect to see the similar for short-selling as well. Furthermore, if these properties hold, then the $\theta_{i}$ boundary coincides with the $i$-th axis iff the Merton line does, again in accordance with the remark in [SS].

- Let us note that if $\theta_{2}=\pi / 2$, then we face the following interesting situation: Once on the $y$-axis, we have $d X=0$ so that $d M=c d t /(1-\mu)$ is absolutely continuous for $t>0$ and we face a pure consumption optimization problem. (A similar thing happens on the $x$-axis 
if $\theta_{1}=0$ ). It is fairly obvious that if the no transaction region has no boundaries coinciding with axes, then $\mathcal{L}$ and $\mathcal{M}$ are $d t$-singular, while they are absolutely continuous (for $t>0$ ) if on the axes. This may be the explanation why it has turned out to be difficult to prove the value function to be $C^{2}$ on the axes.

- In all cases, we conjecture that the assumptions made to ensure that the Merton line lies in the first quadrant, are not needed.

Acknowledgements. We wish to thank P. Protter for helpful comments. N.C. Framstad acknowledges financial support from the Norwegian Research Council and NORFA. Parts of this work were carried out while N.C. Framstad visited the Stockholm School of Economics and B. Øksendal visited the University of California at Irvine. We want to thank these institutions for their hospitality.

\section{References}

[A] K.K. Aase: Optimum portfolio diversification in a general continuous-time model. Stochastic Process. Appl. 18 (1984), 81-98.

[AMS] M. Akian, J.L. Menaldi and A. Sulem: On an investment-consumption model with transaction costs. SIAM J. Control Opt. 34 (1996), 329-364.

[AST] M. Akian, A. Sulem and M.I. Taksar: Ergodic multidimensional diffusion dynamic portfolio selection model. Research Report INRIA 3626, February 1999.

[BL] A. Bensoussan and J.-L. Lions: Impulse Control and Quasi-Variational Inequalities. Gauthier-Villars 1984.

[CEM] M. Chaleyat-Maurel, N. El Karoui and B. Marchal: Reflexion discontinue et systemes stochastiques. Ann. Prob. 8 (1980), 1049-1067.

[DN] M.H.A. Davis and A. Norman: Portfolio selection with transaction costs. Math. Oper. Res. 15 (1990), 676-713.

[F] N.C. Framstad: Combined Stochastic Control for Jump Diffusions with Applications to Economics. Cand. Scient. Thesis University of Oslo Dec. 1997.

[FØS] N.C. Framstad, B. Øksendal and A. Sulem: Optimal consumption and portfolio in a jump diffusion market. In A. Shiryaev and A. Sulem (editors): Workshop on Mathematical Finance. INRIA, Paris 1998, and Discussion paper 5/99, March 1999, ISSN:15004066, Norwegian School of Economics and Business Administration, Helleveien 30,N-5035 Bergen-Sandviken, Norway.

[JS] J. Jacod and A. Shiryaev: Limit Theorems for Stochastic Processes. Springer-Verlag 1987.

[Ph] H. Pham: Optimal stopping of controlled jump diffusion processes: A viscosity solution approach. J. Math. Systems, Estimation and Control 8 (1998), 1-27.

[P] P. Protter: Stochastic Integration and Differential Equations. Springer-Verlag 1990.

[R] R.T. Rockafeller: Convex Analysis. Princeton Univ. Press 1970. 
[S] S.E. Shreve: Liquidity premium for capital asset pricing with transaction costs. In M.H.A. Davis et al. (editors): Mathematical Finance (IMA vol. 65), Springer 1995.

[SS] S.E. Shreve and H.M. Soner: Optimal investment and consumption with transaction costs. Ann. Appl. Prob. 4 (1994), 609-692.

[Z] T. Zhang: Optimal time to invest when the price processes are of jump type. Manuscript, Haugesund/Stord College 1998. 
Unit'e de recherche INRIA Lorraine, Technopôle de Nancy-Brabois, Campus scientifique, 615 rue du Jardin Botanique, BP 101, 54600 VILLERS LÈS NANCY

Unit'e de recherche INRIA Rennes, Irisa, Campus universitaire de Beaulieu, 35042 RENNES Cedex Unit'e de recherche INRIA Rhône-Alpes, 655, avenue de l'Europe, 38330 MONTBONNOT ST MARTIN

Unit'e de recherche INRIA Rocquencourt, Domaine de Voluceau, Rocquencourt, BP 105, 78153 LE CHESNAY Cedex

Unit'e de recherche INRIA Sophia-Antipolis, 2004 route des Lucioles, BP 93, 06902 SOPHIA-ANTIPOLIS Cedex

\section{Éditeur}

INRIA, Domaine de Voluceau, Rocquencourt, BP 105, 78153 LE CHESNAY Cedex (France)

http://www.inria.fr

ISSN 0249-6399 